\title{
Frecuencia de casos de Malaria y los factores contribuyentes en el distrito de Huepetuhe, Madre de Dios, Perú
}

\author{
Frequency of and risk factors for malaria in the District of Huepetuhe, Madre de Dios, Peru.
}

\author{
Jesús Rojas Jaimes ${ }^{1}$
}

RESUMEN

Objetivos: Determinar la frecuencia de casos de Malaria en pacientes febriles y los factores condicionantes en el distrito de Huepetuhe, Madre de Dios. Material y métodos: Estudio descriptivo y transversal. Se incluyeron 170 pacientes febriles atendidos en el Laboratorio del Centro de Salud de Huepetuhe. Se utilizó el examen de la gota gruesa y la prueba rápida de cromatografía, para el diagnóstico y tipificación del Plasmodium. Se realizaron visitas de campo para evaluar la existencia de condiciones epidemiológicas que favorecen el desarrollo de la malaria. Resultados: Se encontraron 124 (72,9\%) pacientes positivos para malaria, todos correspondieron a P. vivax. El 75\% fue varón y 64,5\% tenía entre 20 y 59 años. Más de la mitad de los pacientes procedía de Alto Puquiri $(27,4 \%)$ y Tranquera (26,6\%). Conclusiones: La malaria es endémica en los centros poblados de Alto Puquiri y Tranquera, zonas periféricas del distrito de Huepetuhe, donde se realiza minería informal.

PALABRAS CLAVE: Malaria, endemia, Plasmodium vivax (Fuente: DeCS BIREME).

\section{SUMMARY}

Objective: To determine the frequency of and risk factors for malaria in the District of Huepetuhe, Madre de Dios, Peru. Methods: Cross sectional study that included 170 febrile patients attended at the Huepetuhe's Peripheral Health Care Centre Laboratory. Thick smears and rapid diagnostic tests were performed to diagnose and speciate Plasmodium parasites. Field visits were carried-out to evaluate risk factors for malaria acquisition. Results: 124 (72.9\%) patients tested positive for malaria, all of them were infected with P. vivax; $75 \%$ were males; $64.5 \%$ had between 20 and 59 years of age. More than half of the patients came from Alto Puquiri (27.4\%) and Tranquera (26.2\%). Conclusions: Malaria is endemic in Alto Puquiri and Tranquera were illegal mining is performed.

KEYWORDS: Malaria, endemic, Plasmodium vivax (Source: MeSH NLM).

\section{INTRODUCCIÓN}

La malaria es una de las pandemias más desbastadoras que hoy en día enfrentamos (1). Esta enfermedad cobra numerosas vidas especialmente en el continente africano. La malaria es causada por un protozoario del género Plasmodium, encontrándose 4 especies que causan enfermedad en el hombre:
Plasmodium vivax, Plasmodium falciparum, Plasmodium ovale y Plasmadium malariae.

En Perú, dos especies son importantes desde el punto de vista de la salud pública, Plasmodium falciparum y Plasmodium vivax. El cuadro clínico es generalmente más severo en la infección por $P$. falciparum, aunque influyen otros factores como

1 Biólogo. Laboratorio de Salud Pública de Huepetuhe, Ministerio de Salud. Madre de Dios, Perú. 
número de veces de infección, estado nutricional del paciente, edad y otras comorbilidades asociadas.

La identificación de la especie es de importancia para la lección de la terapia. Existen varias técnicas para poder identificar infección por Plasmodium sp desde las técnicas básicas no complicadas y baratas como la microscopia, aunque de baja especificidad cuando la persona no está bien entrenada, hasta las técnicas moleculares que son muy eficientes en especificidad y sensibilidad, aunque el costo es elevado (2).

En el Perú se atribuye la mayor cantidad de casos de malaria al Plasmodium vivax con una supuesta presencia de malaria no complicada, aunque las formas complicadas se podrán observar cuando exista comorbilidad de otros agentes como es en el caso de algunos lugares en el país (3).

La malaria es una enfermedad que debe ser enfocada de manera integral considerando múltiples agentes que contribuyen a la enfermedad como el medio ambiente, la genética del paciente, el agente etiológico y el estilo de vida de la población, para el diseño e implementación de la estrategia para la erradicación de la enfermedad por los órganos competentes, siendo actualmente la política asistencial y farmacológica correcta, aunque limitada (4).

El objetivo del estudio fue determinar la frecuencia de casos de malaria en pacientes febriles y los factores condicionantes en el distrito de Huepetuhe, Madre de Dios.

\section{MATERIAL Y MÉTODOS}

Estudio descriptivo, transversal, realizado en el laboratorio de Salud Pública de Huepetuhe, Madre de Dios, Perú. Se incluyeron 170 pacientes febriles, atendidos en el laboratorio entre el 4 de enero y el 27 de marzo de 2011.

El distrito de Huepetuhe, pertenece a la provincia de Manu, es un distrito joven con mucha gente migrante especialmente de Cusco, Puno y Arequipa que se dedican a la minería aurífera informal. El distrito consta de un centro poblado céntrico en donde no se cuenta con reportes de infecciones de malaria autóctona (datos epidemiológicos no publicados). Los centros poblados periféricos de condiciones rurales, en plena selva baja donde se encuentran los campamentos es donde se reportan los casos de malaria.
Para el diagnóstico se utilizó el examen de la gota gruesa, para ello se obtuvo 2 gotas de sangre por punción en el dedo; en una gota se realizó un extendido elíptico y en la otra un extendido de la mitad del portaobjeto hacia abajo. Luego de secado, se fijó con etanol al 96\% y se procedió con la tinción Giemsa por 10 minutos, paso seguido, se realizó la lectura en el microscopio a 1000X para identificar al parásito.

Para la identificación de la especie, se utilizó el método de cromatografía. Se colocó una gota de sangre en el pocillo de la muestra, se agregaron las gotas del buffer de corrida, procediendo a la lectura en 15 minutos utilizando dos marcas de pruebas rápidas de cromatografía: Bioline ${ }^{\circledR}$ y Care Start ${ }^{\circledR}$. La lectura consistió en ver la banda control, que debe aparecer siempre para ser la prueba válida, y los resultados de las bandas 1 y/o 2. La banda 1 identifica todo el complejo de Plasmodium sp y la banda 2 es específica para Plasmodium falciparum.

Se realizaron visitas de campo a las localidades de Barranco Chico-Tranquera y Alto Puquiri, lugares de mayor endemia de malaria en el distrito de Huepetuhe, para evaluar las condiciones epidemiológicas que favorecen el desarrollo de la malaria.

\section{Análisis de datos}

Se utilizó estadística descriptiva; se determinaron frecuencias y porcentajes. Se utilizó el Programa Microsoft Excel para Windows.

\section{RESULTADOS}

Se encontraron $124 \quad(72,9 \%)$ pacientes con gota gruesa y cromatografía positivas; todos correspondieron a Plasmodium vivax. El 75\% fueron varones. Ochenta $(64,5 \%)$ tenían entre 20 y 59 años, $32(25,8 \%)$ entre 10 y 19 años, $11(8,9 \%)$ menores de 10 años y uno, 60 años a más.

Más de la mitad de los pacientes procedía de Alto Puquiri $(27,4 \%)$ y Tranquera (26,6\%) (Gráfico 1 y figura 1).

Durante la visita a las localidades de alta frecuencia de malaria se encontró la presencia y creación de pozos artificiales que sirven de criaderos de vectores, presencia de desnutrición crónica, de parasitosis, contaminación por mercurio, la venta de antimaláricos y automedicación, y alta tasa de migración por la búsqueda de oportunidades laborales. 


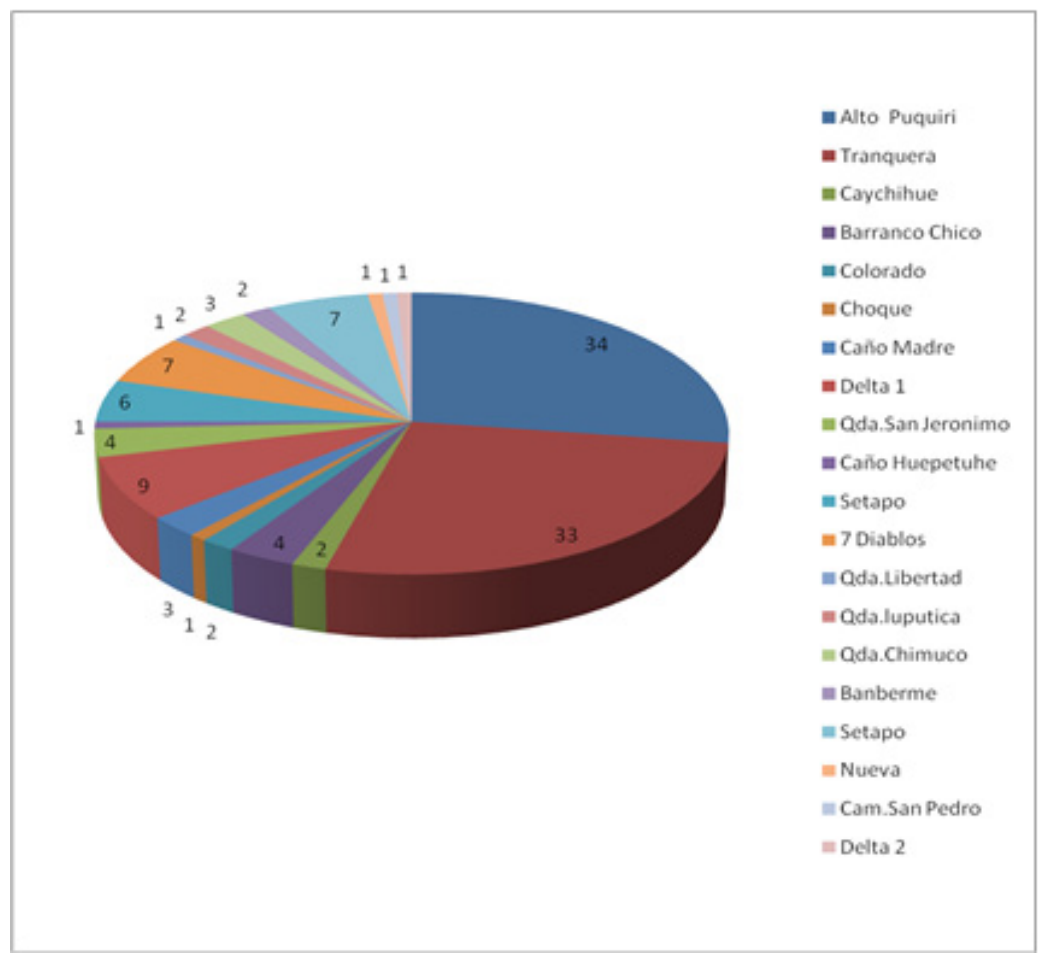

Gráfico 1. Procedencia de los casos de Malaria.

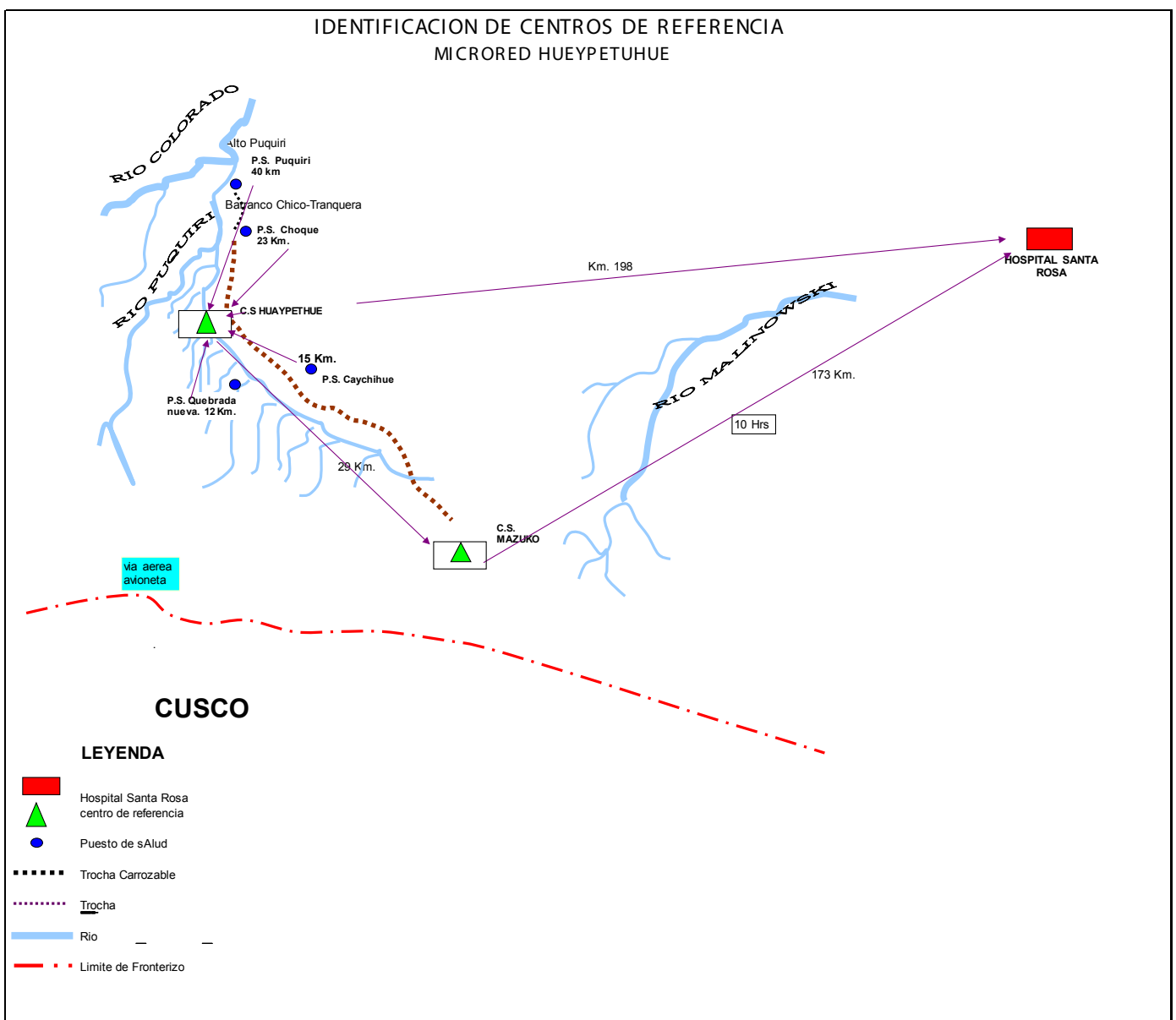

Figura 1. Mapa geográfico de la jurisdicción de la microred Huepetuhe y los sitios endémicos Tranquera y Alto Puquiri 


\section{DISCUSIÓN}

Todos los casos de Malaria fueron causados por Plasmodium vivax, de tal manera que la terapia que se brinda en este lugar con cloroquina y primaquina según el esquema nacional es adecuada; pero se deberían evaluar terapias con menos efectos colaterales y el uso de la quimioprofilaxis con cloroquina que es el antimalárico de elección en la fase circulante del parásito, y que su uso ha demostrado buenos resultados $(5,6)$.

El cuadro clínico fue de moderada severidad, aunque existió un caso de malaria grave que presentó hemorragia digestiva alta. El cuadro clínico se caracterizó por las tembladeras, la fiebre intermitente (7), el dolor abdominal y la ictericia en los casos más complicados.

La mayoría de las personas infectadas fueron hombres, adultos jóvenes y adolescentes (entre 10 y 59 años); esto debido a que constituyen la fuerza laboral de los campamentos mineros. Más de la mitad de los casos provenía de las localidades de Alto Puquiri y Tranquera, dos localidades periféricas al centro poblado (Figura 1), donde se desarrolla intensa minería informal para la extracción de oro, donde los mineros construyen inmensos pozos para extraer agua, los cuales no son rellenados después de utilizarlos convirtiéndose en criaderos del Anopheles sp, vector del Plasmodium $s p$.

En el estudio de campo se encontraron factores que favorecen la endemia, entre ellas, los estilos de vida de la población, la alta migración a las zonas endémicas de malaria de poblaciones no expuestas previamente al patógeno (8), la construcción de pozos inmensos que sirven de criaderos del vector y el hecho que muchos de los campamentos están cerca al rio Huepetuhe. Además, otras situaciones que afectan la salud como la mala nutrición, parasitosis intestinal, anemia, procesos infecciosos y riesgo de toxicidad por el uso del mercurio.

Otro factor de riesgo es la venta informal de medicamentos en el distrito de Huepetuhe, sin garantía y en muchos casos con dosificación incorrecta lo que incrementa el riesgo de resistencia a los antimaláricos.

Es de suma importancia la implementación y ejecución correcta de los programas de salud promocional y preventiva y aplicar sanciones a quienes incurran en actos que atenten contra la salud como la realización de pozos que se convierten en criaderos de vectores $(8,9)$.

De realizarse acciones de poco alcance y mal planificadas, serán similares a las que hasta el momento se vienen realizando, es decir, meramente asistenciales y limitadas, con aumento de la morbilidad y mortalidad, y con un gasto publico fuerte por la alta demanda de antimaláricos y de personal. Adicionalmente, la aplicación de una buena política de conservación ambiental que frene la tala de los árboles y deforestación indiscriminada, como viene ocurriendo en Huepetuhe, que al dejar el suelo árido e infértil incrementa la evaporación con el aumento consecuente de las precipitaciones y temperatura, favoreciendo la proliferación de los vectores, como el de la malaria (10).

Las medidas inmediatas recomendadas para bajar la morbilidad por malaria son: Exigir a los mineros informales el relleno de los pozos, a través de una Ordenanza de Alcaldía; la fumigación intensiva en los lugares endémicos previa evaluación de susceptibilidad entomológica al insecticida; implementación y ejecución de los programas de salud promocional y preventivo por medio de los profesionales de la salud, y realizar planes integrales de intervención diagnóstica y farmacológica en campo en las zonas endémicas, usando las pruebas rápidas que resultan muy útiles en estas circunstancias (11).

Se concluye que la malaria es endémica en los centros poblados de Alto Puquiri y Tranquera, zonas periféricas del distrito de Huepetuhe, donde se realiza minería informal.

\section{Declaración de financiamiento y de conflictos de intereses:}

El trabajo fue financiado por el autor, quien declara no tener conflictos de intereses.

\section{Correspondencia:}

Jesús Rojas Jaimes

Av.Cerro de Pasco 319-Comas.

Lima, Perú.

Correo electrónico: fago200179@yahoo.es

\section{REFERENCIAS BIBLIOGRÁFICAS}

1. World Health Organization. World Malaria Report. Washington: WHO; 2009. 
2. Marangi M, Di Tullio R, Mens P, et al. Prevalence of Plasmodium spp. in malaria asymptomatic African migrants assessed by nucleic acid sequence based amplification. Malaria Journal. 2009; 8:12. doi:10.1186/1475-2875-8-12

3. Legua P. Malaria en el Perú. Rev Med Hered. 1994; 5(3): 115-17.

4. Widmer L, Blank P, Van Herck K, Hatz1 C, Schlagenhauf1 P. Cost-effectiveness analysis of malaria chemoprophylaxis for travellers to WestAfrica. BMC Infectious Diseases. 2010; 279(10):110.

5. Daneshvar C, Davis T, Cox-Singh J, et al. Clinical and parasitological response to oral chloroquine and primaquine in uncomplicated human Plasmodium knowlesi infections. Malaria Journal. 2010; 9: 238. doi:10.1186/1475-2875-9-238.

6. Poravuth Y, Scheat D, Rueangweerayut R. Pyronaridine-artesunate versus chloroquine in patients with acute Plasmodium vivax malaria: A randomized, double-blind, non-inferiority trial. PLoS One. 2011; 6(1):1-12.
7. Ruiz W, Tokeshi G, Shirota A, Pichilingue O, Prieto O. Malaria por Plasmodium vivax: Aspectos clínicos y hematológicos. Rev Med Hered. 1994; 5; 118-128.

8. Elyazar I, Hay S, Baird K. Malaria distribution, prevalence, drug resistance and control in Indonesia. Adv Parasitol. 2011; 74(41): 1-107.

9. Cibulskis R, Aregawi M, Williams R, Otten M, Dye C. Worldwide incidence of Malaria in 2009: Estimates, time trends and a critique of methods. PLoS Medicine. 2011; 8(12): 1-12.

10. Xiao D, Long Y, Wang S, et al. Spatiotemporal distribution of malaria and the association between its epidemic and climate factors in Hainan, China. Malaria Journal. 2010; 9:185.

11. Arrospide N, Flores R, Ruiz J. Evaluación de una prueba rápida para el diagnóstico de malaria en áreas endémicas del Perú. Rev Peru Med Exp Salud Publica. 2006; 23(2): 81-86.

Recibido: 10/07/2012 Aceptado: 20/05/2013 